\title{
Anabases
}

ANABASES Traditions et réceptions de l'Antiquité

$7 \mid 2008$

Varia

\section{Régénération et dégénérescence : la philosophie grecque reçue et relue par les nazis (Platon et la Stoa)}

Johann Chapoutot

\section{CpenEdition}

Journals

Édition électronique

URL : http://journals.openedition.org/anabases/2510

DOI : 10.4000/anabases. 2510

ISSN : 2256-9421

Éditeur

E.R.A.S.M.E.

\section{Édition imprimée}

Date de publication : 1 mars 2008

Pagination : 141-161

ISSN : $1774-4296$

Référence électronique

Johann Chapoutot, «Régénération et dégénérescence : la philosophie grecque reçue et relue par les nazis (Platon et la Stoa) », Anabases [En ligne], 7 | 2008, mis en ligne le 01 décembre 2011, consulté le 14 novembre 2019. URL : http://journals.openedition.org/anabases/2510 
Anabases 7 (2008), p. 141-161.

\section{Régénération et dégénérescence : la philosophie grecque reçue et relue par les nazis (Platon et la Stoa)}

JOHANN CHAPOUTOT

LA PHILOSOPHIE GRECQUE est « une des créations spirituelles les plus importantes qui soient issues du sol de la race et du peuple indogermanique, c'est-à-dire nordique ${ }^{1}$ ". Voilà ce que l'on peut lire, en 1943, dans l'édition revue du dictionnaire philosophique de référence des étudiants allemands, le Philosophisches Wörterbuch de Schmidt. Au cours d'une conférence prononcée durant l'été 1941, dans l'ivresse de victoires sans fin et d'un Blitzkrieg balkanique foudroyant, l'historien de la philosophie Oskar Becker se fait plus explicite : «La philosophie grecque est la philosophie d'un peuple qui nous est apparenté par la race ${ }^{2}$."

1 "Griechische Philosophie", in H. SCHMIDT, Philosophisches Wörterbuch - Zehnte Auflage, völlig neu bearbeitet von Werner Schingnitz und Joachim Schondorff, Stuttgart, Alfred Kröner Verlag, 1943, 653 p., p. 208. La 9e et dernière édition du vivant de l'auteur datait de 1934. L'avant-propos de la dixième édition, en 1943, précise que la philosophie ne pouvait faire l'économie d'un aggiornamento à la suite de la révolution nationale-socialiste : "Le temps n'est pas immobile. De nouveaux noms, de nouveaux concepts apparaissent, d'autres disparaissent, de nouvelles acceptions et valeurs s'imposent et font apparaître sous un jour nouveau non seulement le présent, mais aussi le passé [...]. La grande révolution des valeurs qui s'est accomplie dans tous les domaines de la vie en Allemagne depuis 1933 concerne aussi la science et avant tout la philosophie qui est depuis toujours concernée, voire entièrement occupée par les questions idéologiques. En conséquence, il fallait plus que jamais que les valeurs de l'idéologie occupassent le premier plan. »

2 O. BECKER, "Die griechische Philosophie", in O. BECKER, K. CHUdOBA, et alii, Griechenland, Bonn, Kriegsvorträge der Rheinischen Friedrich-Wilhelm-Universität Wissenschaft im Kampf für Deutschland, 1944, 522 p., p. 241-267, p. 242. 
Deux éléments sont à noter dans les citations qui précèdent. Le premier est que les nazis, quand ils entendent le mot culture, ne sortent pas systématiquement leur revolver, une phrase attribuée à Goebbels et parfaitement apocryphe. Le second est cette curieuse juxtaposition des mots " race " et "philosophie " qui, à nos yeux, frise l'oxymore. Le mot "race ", ainsi que ses connexes, fait l'objet de longs développements dans la nouvelle édition du dictionnaire de Schmidt. Cette notion aux contours tellement flous que des intellectuels du Parti nazi s'étaient abondamment, et jusque dans les années 1930, traités de charlatans à son sujet, est érigée en concept herméneutique cardinal.

Quand ils entendent le mot culture, les nazis sortent donc plutôt leur stéthoscope, ou leur craniomètre. Le racisme national-socialiste, par le déterminisme biologique strict qu'il défend, induit une appréhension médicale de toute création humaine, une conception symptomatologique des œuvres de culture.

On constate que, de même qu'elle opère une biologisation du politique, la doctrine nationale-socialiste effectue une naturalisation du culturel. Une œuvre de culture, fût-elle cathédrale médiévale, peinture de la Renaissance ou traité de philosophie grecque, n'est pas le produit de l'inspiration ou du libre-arbitre d'un individu, moins encore, pour ce qui concerne le cas spécifique de la philosophie, d'une prétendue raison universelle, chimère libérale et cosmopolite que les nazis répudient. Aucune œuvre de l'esprit n'échappe au déterminisme du sang et du sol : Blut und Boden sont les conditions nécessitantes de la création humaine. Le biologisme déterministe du racisme nazi opère ainsi une réduction de l'idéologie à la biologie, et, plus généralement, du culturel au naturel. Il promeut une sorte d'hégélianisme incarnant qui voit dans toute production culturelle non pas une réification de l'esprit, mais une matérialisation de ce sang dont l'esprit n'est qu'une manifestation, qu'une expression, une modalité d'être. Le sang, et les qualités de ce sang, décident de tout.

Le rapport des nazis à l'art en témoigne. L'art expressionniste allemand, selon le Professeur Paul Schultze-Naumburg, est ainsi l'expression d'une santé affectée, d'un corps et d'un esprit à ce point malades que des barbouilleurs en viennent à peindre des chevaux bleus. Dans son instructif Kunst und Rasse ("L'art et la race ») publié en 1928, Schultze-Naumburg défend l'idée que si Franz Marc et d'autres peignent ainsi, c'est qu'ils voient effectivement les chevaux en bleu ou les arbres en rouge. Leur art n'est que l'expression fidèle d'une biologie morbide, d'un système nerveux défaillant, d'une perception sensible irrémédiablement affectée. Il convient dès lors de considérer leurs œuvres en raciologues 3 éclairés, non comme de l'art, objet d'une jouissance désintéressée, mais comme les symptômes d'une pathologie, objet d'une qualification nosographique. Après en avoir interdit l'accès au public, qu'il faut au contraire édifier par la représentation d'une saine beauté et protéger des expressions du malsain, le raciologue 
étudiera ces manifestations morbides et en proposera une raisonnable étiologie : le détraquement du système nerveux qui a abouti à de telles incongruités est dû à un mélange racial ou à une affection dont il faut protéger la race en éloignant le sujet morbide et ses œuvres.

On comprend donc que l'adjectif "dégénéré » dans l'expression consacrée d' " art dégénéré " est loin d'être une simple clause de style, une épithète ornementale ou benoîtement polémique. Plus profondément qu'une simple insulte, ce mot exprime une répudiation biologique, un jugement médical plus que négatif, annihilant, car il est ici à comprendre au sens littéral : l'art condamné par les nazis est l'expression d'un sang dé-généré, c'est-à-dire qui a déchu de son genre, de son espèce. L'Allemand parle d'entartete Kunst, ent-artet signifiant privé de sa Art, mot allemand synonyme de Rasse.

Cette herméneutique biologique et médicale, cette lecture raciologique de l'art est également mobilisée pour d'autres réalités culturelles, comme la philosophie grecque : une œuvre philosophique, malgré qu'elle en ait, n'est pas l'expression abstraite d'une idéalité absolue. Elle est incarnée, fille de son temps, du sol et du sang. Avant la rédaction de Mein Kampf par Hitler (1924) ou du Mythe du vingtième siècle par Alfred Rosenberg (1935), l'idéalité philosophique, cette prétention sinon à l'absolu, du moins au général, avait déjà été interrogée par les philosophies du soupçon, qui en avaient montré toute la relativité à un temps, à un lieu, voire à une idiosyncrasie physique : Marx avait référé la pseudo-universalité philosophique à ses conditions de production socio-économiques, Nietzsche avait disserté sur le " problème de Socrate ", cette idiosyncrasie du raté qui se voue par ressentiment à la dialectique, et Freud avait rendu le Moi et la Raison du sujet victorien plus humbles face aux puissances formidables du ça. Les nazis participent pleinement de cette démarche du soupçon adressé à la raison, d'autant plus que son règne est solidaire du cosmopolitisme libéral et délétère imposé par la Révolution Française et l'idéologie des Droits de l'Homme. Curieusement, c'est de Freud que, sans oublier Nietzsche, les nazis sont les plus proches. Eux aussi développent une exégèse psychophysique des œuvres de l'esprit, à la réserve, d'importance, que ni Freud, ni Nietzsche ne lient l'expression de la pensée ou de la création artistique à un quelconque déterminisme racial : le complexe d'EEdipe, pour le dire vite, est universel, de même que le travail des forces réactives pour Nietzsche. Ce dernier, d'ailleurs, n'a jamais conçu le surhomme en termes raciaux. La typologie et l'axiologie nietzschéennes n’ont jamais été racistes ni même adossées à une quelconque raciologie, puisque les hommes du ressentiment, les ratés, peuvent être nordiques de même qu'une belle figure du surhomme est offerte par le guerrier juif de l'Ancien Testament.

Dans leur rapport à la philosophie grecque, les nazis, en raison de leur biologisme foncier, opèrent une réduction symptomale. La philosophie révèle ce qu'il en est des Grecs en 530, 399 et 364 avant notre ère, ou plutôt ce qu'il en est du Volkstum grec. Or le destin du Volkstum grec importe aux nazis, comme en témoigne l'abondante littérature historique, anthropologique, politologique, qu'elle soit savante ou de vulgarisation, qui lui est consacrée. Nous avons pu montrer ailleurs toute l'importance que les 
nazis accordent à l'annexion de la Grèce antique à la race nordique ${ }^{4}:$ la vulgate promue par le Parti, puis par le Parti-État est que les Grecs sont de race germanique-nordique, que les cités grecques ont été fondées par des tribus de paysans-soldats issues du nord. Cette annexion de morts glorieux permet de revendiquer, pour le compte d'une race sinon singulièrement pauvre en témoignages de grandeur historique, le prestige d'une culture qui fascine le Bildungsbürgertum allemand depuis Winckelmann, et l'Europe depuis Missolonghi. Les Grecs ont été grands, sublimes, puis ont disparu de la grande scène historique du monde. Le destin de la Grèce ancienne est donc source d'enseignements pour l'Allemagne nouvelle : sa grandeur pourra emprunter les chemins déjà foulés par un grand peuple nordique de l'Antiquité. Sa décadence pourra être évitée si de justes leçons sont tirées de la mort des Grecs en tant que civilisation hégémonique.

Pour étudier le destin du Volkstum grec, il faut se pencher sur son histoire, mais une table chronologique, avec sa succession de dates, apprendra peu de choses si aucune attention n'est accordée à la vision du monde que les Grecs développaient. Leur Weltanschauung a été la condition de leur ascension, son altération, celle de leur chute. La condition et non la cause, puisque la Weltanschauung est elle-même conditionnée par la qualité du sang qui engendrera des idées différentes selon qu'il est pur ou altéré.

La philosophie exprime ainsi un état de la race : le logos n'est rien d'autre que la voix de l'ethnos et de son statut racial. La philosophie est-elle vigoureuse, décisionniste, volontaire qu'elle exprime la vigueur du sang ou sa régénération. Est-elle, au contraire, individualiste ou pessimiste qu'elle exprime sa dégénérescence, sa lente descente vers le marais du mélange et de l'indifférenciation. Si elle est héroïque et aristocratique, elle exprime avec certitude la pureté d'un sang nordique encore immaculé. Si, au contraire, elle est démocratique et égalitariste, elle trahit le mélange d'un sang perdu par la compromission avec d'autres races.

Dans ces conditions, l'étude de la philosophie grecque n'est pas une question réservée à la pieuse érudition des Seminare de Kiel, Bonn ou Heidelberg, ou circonscrite au pâle silence des bibliothèques. Rien ne serait d'ailleurs plus contraire à l'esprit, indubitablement nordique, d'une civilisation qui a promu la kalokagathia, l'idéal de l'homme beau et bon, athlète de l'esprit comme du corps. On comprend mieux, dès lors, pourquoi tant de monde, sous le Troisième Reich, parle de Grèce et de philosophie grecque : Hitler, dans Mein Kampf comme dans nombre de ses discours, Alfred Rosenberg, Joseph Goebbels, mais aussi Goering et Himmler. Spécialistes de lettres classiques et d'histoire ancienne inaugurent un revival de Platon et de Sparte, fixant un canon pour le récit du destin grec, que reprennent manuels scolaires et fascicules de

4

C'est le propos de notre thèse, Le national-socialisme et l'Antiquité, soutenue à Paris I et à la TU Berlin le 1er décembre 2006, devant un jury composé des professeurs Étienne François et Robert Frank (directeurs), François Hartog, Pascal Ory, Michael Werner et Jakob Vogel. Le texte de cette thèse est sous presse aux Presses Universitaires de France (septembre 2008). 
formation idéologique du Parti et de ses organes. Dans ce récit, Platon occupe une place privilégiée : il est le terminus a quo de la pensée nordique-germanique grecque, premier terme d'une histoire de la Weltanschaunng hellénique dont le terminus ad quem est la dissolution, l'altération définitive de l'idéal aristocratique nordique dans la pensée de la Stoa.

Dans la réception et l'exégèse nazie, le centre de gravité du corpus platonicien se déplace : l'accent ne porte plus, comme généralement depuis les XVIe et XVIII siècles, sur les textes épistémologiques ou gnoséologiques du penseur athénien, mais sur ses textes politiques. Le Platon métaphysicien et théoricien des Idées fut aux yeux des nazis l'étendard d'un humanisme désincarné et d'une Aufklärung au rationalisme hypertrophié. Le théoricien de la connaissance s'efface derrière le penseur de la cité idéale et de la régénération de la communauté.

Dans un fascicule édité par l'association des enseignants nationaux-socialistes (Nationalsozialistischer Lehrerbund, NSLB) à l'attention des professeurs de Grec, le grand helléniste Hans Bogner invite à considérer que le cœur de l'œuvre de Platon est constitué par le triptyque de la "Politeia, du Politikos, du Nomos 5 ", soit de la République, du Politique et des Lois. Le raciologue en chef du Parti nazi Hans Günther, qui a luimême consacré un opus à Platon, adjoint les Lettres 6 à ce corpus. Pour Günther, le Platon qui importe désormais est bel et bien le Platon "penseur de l'État ${ }^{7}$ ", et non le "représentant de la théorie des Idées 8 ": Platon n'est pas un simple savant, il « est de la lignée des Solon et des Clisthène. C'est l'histoire d'Athènes, ce sont ses grands législateurs et hommes d'État [...] qui constituent l'arrière-plan de son œuvre", et non "les

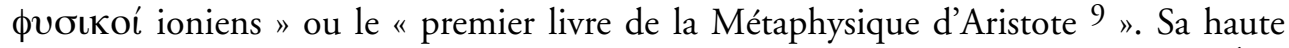
figure est donc plus à sa place dans le Panthéon des législateurs et des hommes d'État que dans celui des simples métaphysiciens. Comme le résume avec force Werner Jaeger à l'occasion d'un article publié en 1933 dans la revue nazie Volk im Werden ("Peuple en devenir "), article où il attaque avec vigueur le XVIII siècle et son humanisme individualiste, «le Platon de notre génération est un créateur d'États, un législateur. Ce n'est plus le systématicien néo-kantien et l'honorable scholarque philosophique que nos prédécesseurs avaient vu en lui 10 ». Kant n'est donc pas, pour Jaeger, l'archétype du

5 H. Bogner, Platon im Unterricht, Auf dem Wege zum nationalpolitischen Gymnasium, Beiträge zur nationalsozialistischen Ausrichtung des altsprachlichen Unterrichts, Reichsfachbearbeiter für alte Sprachen im NSLB, Heft 1, Frankfurt-am-Main, Verlag Moritz Diesterweg, 1937, 39 p., p. 8.

6 H. GÜNTHER, Platon als Hüter des Lebens. Platons Zucht-und Erziehungsgedanken und deren Bedeutung für die Gegenwart, München, Lehmann Verlag, 1928, seconde édition 1935, 80 p., avant-propos.

7 Ibid., p. 16.

$8 \quad$ Ibid.

$9 \quad$ Ibid.

10 W. JAEGER, "Die Erziehung des politischen Menschen und die Antike", in Volk im Werden, I, 3, 1933, p. 43-49, p. 46. 
génie allemand, ce Beethoven de la philosophie que l'on célèbre parfois. Il est le dangereux avatar de Lumières cosmopolites et désincarnées. Le prétendu idéalisme platonicien est, au contraire, un réalisme rude et lucide, dont, selon le philosophe et spécialiste de Platon Kurt Hildebrandt, «seul un lecteur superficiel peut dire qu'il s'agit d'un inaccessible et céleste idéal kantien ${ }^{11}$ ».

Les nazis et leurs séides du monde pédagogique et universitaire s'arment d'une plume virile pour rejeter vigoureusement la figure hâve et chétive du théoricien anémié, du ratiocinant entré en philosophie comme on entre en religion, ayant voué sa vie à l'étude des abstractions au détriment des concrétudes du réel. Hitler, dans Mein Kampf, n'a pas de mots assez durs pour fustiger ce qui constitue, à ses yeux, un dévoiement de l'idéal grec par le Gymnasium allemand. Bien loin de former le kalos k'agathos de l'éducation antique, il atrophie les corps au bénéfice d'une mégacéphalie dégénérée.

Or, Platon apparaît comme l'antithèse achevée de ce type honni. Bien loin d'être un savant rachitique, un homme de cabinet, il est pour Hans Günther l'accomplissement de "l'homme complet 12 ", à la fois penseur puissant, sportif émérite et guerrier achevé. Rien d'étonnant quand on songe, comme le rappelle Günther, que Platon était un homme nordique, issu « de la plus haute noblesse de l'Attique $[\ldots]$ où, jusqu'à une époque tardive, le sang nordique de l'hellénité originelle s'est le mieux conservé à Athènes 13 ». Platon est donc l'incarnation de cet homme nordique accompli, qui a porté à leur plein développement les talents physiques et intellectuels dont sa race recelait les germes.

Günther souligne que Platon n'est pas seulement ce « simple logicien ou théoricien de la connaissance " que les "professeurs de philosophie 14 ", ces incorrigibles perpétrateurs d'abstraction rationnelle, en ont fait. Tout au long de son ouvrage, il ne cesse de prendre à partie les Philosophieprofessoren, figures de la démission face à la vie et du renoncement racial, qu'il oppose à l'archétype du philosophe grec.

Cette nouvelle exégèse de l'opus platonicien n'est pas circonscrite au champ clos des joutes académiques, où s'ébrouent désormais avec bonheur les partisans d'une grécité nordique. Platon est également un objet d'enseignement pour les classes d'histoire et de grec du lycée.

Qu'il faille porter Platon à la connaissance des lycéens du IIIe Reich est une évidence pour platoniciens et hellénistes : pour Adolf Rusch, professeur de lettres classiques du Lycée Mommsen de Berlin-Grunewald, Platon n'est rien moins que « l'édu-

K. Hildebrandt, Platon. Der Kampf des Geistes um die Macht, Berlin, Georg Bondi, 1933, 400 p., p. 265.

Ibid., p. 9.

Ibid., p. 11. 
cateur de l'homme allemand 15 ». Dans un article qu'il consacre à l'enseignement de Platon, Rusch rappelle que l'école nouvelle doit oublier l'individu pour privilégier le groupe, la communauté du peuple, la Volksgemeinschaft. Or Platon est l'homme qui a mené le combat contre "la sophistique ", cette expression malheureuse d'un "individualisme outrancier ". Contre " la maxime selon laquelle l'homme serait la mesure de toute chose " qui rendit Protagoras célèbre, Platon replace l'individu " dans le tout de l'ordre du monde ", dans l'ordre du cosmos comme dans celui de la polis. Platon est donc, "pour l'éducation du jeune homme dans l'État qui est le nôtre aujourd'hui et pour l'État qui est le nôtre ", " le plus sûr moyen 16 " de parvenir à susciter une génération nouvelle, dévouée au corps du peuple et à l'État.

À lire les manuels scolaires, on constate que le Platon du cours d'histoire est présenté comme une haute figure de la résistance nordique à la décadence raciale, intellectuelle et morale de la cité athénienne et du monde grec en général, miné, malgré les objurgations du philosophe, par le mal qui devait l'emporter. Le cours de Grec présente lui aussi en Platon le dernier sursaut de l'hellénité nordique. En 1936, dans le cadre de consultations organisées par Bernhard Rust, professeur de latin, nazi de la première heure et ministre de l'Éducation du Reich, en vue d'une réforme des programmes de l'enseignement secondaire, un mémoire rédigé par un enseignant de lettres classiques et conservé aux Archives fédérales de Berlin-Lichterfelde, fait ainsi l'article du philosophe : «Il faut enseigner Platon aux élèves, précisément parce qu'avec lui se dresse l'esprit nordique dans un combat contre la décadence la plus délétère, contre toute une époque de destruction. Même s'il ne put pas empêcher la dégénérescence du peuple athénien, son combat résonne encore de nos jours chez tous les hommes appartenant à sa race ${ }^{17}$."

À tous ceux qui s'aviseraient de ne voir en Platon qu'un esprit éthéré voguant dans le ciel diaphane des Idées, ce mémorandum adressé par un enseignant au ministère, oppose que, bien au contraire, "l'esprit actif nordique a trouvé sa plus sublime expression dans la philosophie de Platon. La quête de la connaissance se trouve chez lui placée au service de la formation des hommes et des États. Si la contemplation est, chez l'oriental, une fin en soi, la contemplation platonicienne n'est, elle, jamais dissociée de l'action 18 ».

15 A. RusCH, "Platon als Erzieher zum deutschen Menschen", in Humanistische Bildung im Nationalsozialistischen Staate, Neue Wege zur Antike, Erste Reihe, Heft 9, Leipzig und Berlin, Teubner Verlag, 1933, 80 p., p. 44-49.

Ibid., p. 45

17 "Vorschläge für die Gestaltung des Unterrichts in den alten Sprachen ", von Oberstudiendirektor Billen, 1936, 26 p., BABL / R/4901/4614, fo 244-269, fo 245-246. L'abréviation BABL désigne les archives fédérales allemandes (Bundesarchiv BerlinLichterfelde).

18 Richtlinien für Griechisch, Mecklemburgisches Staatsministerium, Abt. Unterricht, BABL / R/4901/4614, fo 279-285, fo 281-282. 
Lintroduction, ici, du couple action/contemplation, traditionnel en philosophie, vise à nier l'image d'un Platon contemplatif ergotant sans fin sous le ciel athénien sur l'immortalité de l'âme et la réalité des Idées. Platon est l'homme de la praxis, de l'action politique, et non de la theoria ou de la contemplatio métaphysique. Celle-ci est résolument subordonnée à celle-là. L'art pour l'art de la contemplation gratuite est une perversion de l'esprit, le retrait du monde d'une race ennemie de la vie et du corps, irréductiblement individualiste et morbide : la race orientale. Le déterminisme racial conditionne l'attitude de Platon comme celle des penseurs contemporains (les sophistes), ou postérieurs (les stoïciens), issus d'Orient : la vita activa, le bios politikos, est éminemment platonicienne, parce que l'esprit nordique est essentiellement engagé, actif 19.

Selon un autre mémoire adressé au ministre Rust, enseigner Platon revient donc à " pratiquer une éducation raciale 20 ». On aura donc soin, en toute bonne pédagogie raciste, de soigneusement opposer à Platon " la sophistique et la rhétorique comme expression d'une influence étrangère à la race » et comme «signe de décadence 21 ». Les nouveaux programmes de 1938, ceux de la réforme Rust, tiennent compte des plaidoyers de ce type. Platon compte au nombre des quatre auteurs inscrits au programme des différentes sections de Grec ${ }^{22}$, avec Homère, Thucydide et Xénophon. Ce dernier est préféré à d'autres classiques de l'enseignement du Grec, comme Isocrate et Démosthène, coupables d'être les figures d'un IVe siècle athénien décadent, tout comme Aristote ou les stoïciens, qui ne sont, ni l'un ni les autres, inscrits au programme de l'enseignement du Grec. Le poids de Platon dans les programmes est renforcé si l'on considère tout ce qu'il y a de platonicien chez Xénophon qui fut à la fois son rival et, comme lui, un philospartiate résolument opposé à la démocratie athénienne.

Avec Platon, c'est à une éthique du combat que les élèves allemands seront éduqués. Günther observe que le mot philosophe, qui n'est plus pour nous « qu'un mot composé de quatre syllabes mortes qui ne disent plus rien qu'à l'érudit 23 ", avait une toute autre signification pour les Grecs.

Kurt Hildebrandt, prévient contre l'erreur qu'il y aurait à confondre le philosophe avec « le savant de cathèdre 24 » ou le « chercheur abstrait 25 ». Le philosophe est un

19 Le Leistungsmensch de la typologie psycho-raciale de Ludwig Clauss s'oppose au Darbietungsmensch oriental, soumis, effacé et sournois. Cf. L. CLAUSS, Rasse und Seele. Eine Einführung in den Sinn der leiblichen Gestalt, Munich, Lehmann Verlag, 1926, $189 \mathrm{p}$.

Richtlinien für Griechisch, Mecklemburgisches Staatsministerium, mémoire cité, fo 281282.

21 Ibid.

22 B. RusT (éd.), Erziehung und Unterricht in der Höheren Schule. Amtliche Ausgabe des Reichs- und preussischen Ministeriums für Wissenschaft, Erziehung und Volksbildung, Berlin, Weidmann, 1938, 265 p., p. 250.

24 Hildebrand, Platon. Der Kampf des Geistes um die Macht, p. 226.

Ibid., p. 247. 
guerrier, et, de fait, remarque-t-il, la sémantique militaire structure une grande partie du discours de Platon : «Le philosophe de Platon est le contraire de l'érudit abstrait : il est l'homme qui s'est affirmé dans la guerre comme dans la conduite des affaires de l'État 26. "On voit donc que la conception agonale du monde propre aux Grecs imprègne toute l'œuvre de ce Grec par excellence qu'est Platon, preuve, s'il en est, de son caractère nordique : "Platon se range à l'avis d'Héraclite : polemos est le père de toute chose ", ce qui entraîne que "l'éducation des guerriers est placée au centre de la politeia 27 ", tant " être véritablement homme signifie pour lui être un guerrier 28 ». Ce que l'on appelle les philosophes-rois sont d'ailleurs originellement et avant tout des guerriers que l'on n'a "tirés de leur caste qu'à l'âge de cinquante ans ", après qu'ils se sont particulièrement distingués dans le service des armes et de l'esprit ${ }^{29}$. La figure du guerrier et la réalité du combat font donc l'objet d'une valorisation d'un adoubement intellectuel et culturel. À la même époque, le philosophe Ernst Krieck, dans sa leçon inaugurale du 10 mai 1933, à Berlin, exhorte les étudiants à être des "soldats politiques ", tandis que le Führer und Rektor der Universität Freiburg Martin Heidegger, reprenant la tripartition platonicienne de La République, définit, aux côtés du service des armes et de celui du travail, le service du savoir.

L’œuvre de Platon est, aux termes de l'exégèse nazie, organiquement guerrière. Fille de la guerre du Péloponnèse et de la crise politique et civique qu'elle provoque à Athènes, solidaire d'une culture grecque d'essence agonale, elle ne borne pas son horizon à un " petit État hellénique sans empire extérieur 30 ». Selon Hildebrandt, Platon ambitionne la constitution de ce qu'il nomme un "Grossgriechenland 31 ", une Grande Grèce qui évoque étrangement la Grande Allemagne, le Grossdeutschland pangermaniste puis nazi. En arrière-plan, et de manière implicite, Hildebrandt évoque le spectre de la Kleinstaaterei grecque et de la discorde civile fratricide, d'une guerre permanente entre cités grecques dont Platon percevait bien qu'elle les mènerait à leur disparition. Cet éclatement politique des cités grecques morcelées en entités rivales est par ailleurs souvent assimilé au fractionnement extrême des États allemands avant l'unification de 1871.

Platon est donc un Kämpfer, qui se bat pour la régénération et la survie de son peuple, sorti exsangue et affaibli de la guerre du Péloponnèse, et menacé de subversion raciale par le mélange avec les races asiatiques : l'hémorragie de Sparte et d'Athènes ouvre les veines grecques au sang étranger. L'œuvre de Platon est ainsi dramatisée, présentée comme un lever d'oriflamme dans un contexte de crise historique majeure. Platon se dresse comme recours dans un contexte marqué par l'urgence historique,

Ibid., p. 226.

Ibid., p. 234.

Ibid., p. 238.

Ibid., p. 240.

Ibid., p. 241.

Ibid., p. 241. 
comme le rappelle Günther, qui fait de Platon la dernière vigie nordique d'un monde qui, contaminé et perverti, est sur le point de s'effondrer.

Le parallèle avec Adolf Hitler s'impose donc, que Joachim Bannes, autre spécialiste de Platon, proclame le plus sérieusement du monde dans le titre d'un fascicule qu'il consacre au maître de l'Académie et à l'œuvre du Führer : Le combat d'Hitler et La République de Platon. Une étude sur le fondement idéologique du mouvement de libération national-socialiste 32 . Bannes, Mein Kampf en main, présente les principes fondamentaux de l'idéologie nazie, avant de parler de la Politeia de Platon, et de proposer une étude comparée des biographies respectives du Führer et du philosophe. Tout ceci lui permet de conclure sur les profondes affinités entre ces deux penseurs et praticiens de l'État. L'historien de la Grèce Hans Bogner, quant à lui, se contente de suggérer l'amalgame entre le Führer de l'Allemagne contemporaine et le Führer de la philosophie athénienne en remarquant que l'enseignement du maittre et les solutions vigoureuses qu'il préconisait pour son temps étaient formulées sur " un ton dictatorial 33 ", ce qui ne s'accorde guère avec la réalité d'un Platon dialogique et questionnant, aux dialogues souvent aporétiques, l'essentiel résidant pour lui dans la question, dans le mouvement de l'esprit qui s'arrache à la certitude pour tenter d'atteindre au vrai. Pour tous les platoniciens que nous avons lus, qui ne reculent devant aucun anachronisme ni aucun amalgame, il ne fait aucun doute que Platon est le théoricien du Führergedanke ${ }^{34}$, du principe d'un Führer sélectionné et racialement irréprochable.

Comme Hitler, Platon est un guerrier nordique qui se bat pour sauver son peuple d'une disparition qui le menace. Telle est la lecture que propose l'helléniste Hans Holtorf de Platon, dans l'introduction à une anthologie de textes de La République qu'il a constituée à l'usage des lycées :

Dans cette époque de profond ébranlement de toutes les valeurs morales, le grand Platon se dresse et mène un combat héroïque contre la dégénérescence de son peuple, contre l'esprit désastreux de destruction absolue [...]. Cette grande figure de sage en appelle à la grandeur de l'âme nordique [...]. Le combat que mène aujourd'hui Adolf Hitler a le même objectif sublime. Les paroles du Führer montrent dans quelle direction l'œuvre de Platon doit nous mener et doit entrer dans les âmes de la jeunesse allemande 35 .

Pour l'historien de la philosophie Hans Heyse, qui consacre, en 1934, son discours d'investiture rectorale à "L'idée de science et l'Université allemande », Platon

J. BANNes, Hitlers Kampf und Platons Staat. Eine Studie über den ideologischen Aufbau der nationalsozialistischen Freiheitsbewegung, Berlin, Walther De Gruyter Verlag, 1933, 21 p. Bogner, Platon im Unterricht, p. 15.

35 H. Holtorf, Platon. Auslese und Bildung der Führer und Wehrmänner. Eine Auslese aus dem Staat, Eclogae Grecolatinae, 73, Berlin, 1934. 
est un modèle à suivre pour tout combattant de l'idée national-socialiste. Le propos de Platon n'est pas l'élaboration de ce mièvre pseudo-idéalisme que "le classicisme et un humanisme dépassé en ont retenu ", mais rien moins que "l'essai d'une totale rénovation de l'être national-hellénique (des nationalhellenischen Wesens) 36 „. Le parallèle avec le Führer n'en reste pas là. Pour opérer la renovatio d'une cité athénienne en passe de disparaitre, Platon a pensé et promu, à en lire nos sources, un ordre politique strictement raciste dont, bien avant le XIXe siècle, il est censé avoir été le père.

Hans Günther, dans sa monographie sur le racisme platonicien, fait ainsi du maître de l'Académie un précurseur de "Gobineau, Mendel et Galton 37 ", de ces hommes dont aucun "n'a jamais méconnu la loi d'airain de l'inégalité " entre les êtres, que l'idéalisme perverti et délétère de 1789 est venu combattre ${ }^{38}$. On porte au crédit de Platon d'avoir pris acte de ce fait indubitable qu'est l'inégalité des hommes : La République enseigne qu'il est trois types d'hommes, appelés selon leurs dons et leur conformation à être des producteurs, des guerriers ou des rois. Platon recourt, pour exposer son propos et désigner ces trois types, à l'allégorie des âmes d'or, d'argent et de bronze, dans laquelle Günther veut voir une évidente métaphore des différences de race. Si les philosophes doivent régner, il est évident que "seuls les hommes au sang pur peuvent philosopher 39 » : «la prédisposition à la philosophie » n'est donc pas une question d'apprentissage ou d'application, mais bien " une question de race 40 ». Elle n'est pas une activité loisible au libre-arbitre et au travail de chacun, mais une vocation biologiquement déterminée. Autant, donc, pour l'universalité de la raison.

Il en découle, selon Hildebrandt, que " l'État dépend de la race pure, de la sélection juste 41 » des philosophes-rois et de l'affectation adéquate de chacun à la fonction que la nature lui a assignée. Hans Bogner s'interroge pareillement : "Qui a le droit de philosopher? [...] Certainement pas des bâtards, mais uniquement des hommes au sang pur (Rep. 535c sqq.) [...] des hommes droits de corps et d'esprit $(536$ b) 42 ." Il en résulte donc bel et bien que "l'aptitude à la philosophie était pour Platon une question de race 43 ». Si cette lecture raciologique et eugénique de Platon n'est pas totalement interdite par le texte lui-même, elle fait violence à $L a$ République qui, si elle parle de " cheptel " et de sélection, n’enferme pas les individus dans le strict déterminisme d'une naissance appelée à conditionner toute leur existence. Le racisme nazi enferme ad

36 H. HeYse, Die Idee der Wissenschaft und die Deutsche Universität, Rektoratsrede zu Königsberg, 4. Dezember 1933, Königsberg, Gräfe und Unzer Verlag, 1933, 15 p.

37 GÜNTHER, Platon als Hüter des Lebens, p. 22.

38 Ibid., p. 20.

39 Ibid., p. 29.

40 Ibid., p. 28.

41 Hildebrandt, Platon. Der Kampf des Geistes um die Macht, p. 260.

42 Bogner, Platon im Unterricht, p. 13.

43 H. Bogner, Die Bildung der politischen Elite, Oldenburg, Stalling Verlag, Schriften an die Nation, 1932, 72 p., p. 36. 
vitam le sujet dans l'orbe étroit de sa race, alors que, chez Platon, tout individu qui en est, par ses qualités propres, jugé digne, est éligible à une entrée dans la caste des guerriers et donc des philosophes-rois.

Pour nos auteurs cependant, le racisme de Platon aboutit logiquement à l'eugénisme, dont il est promu théoricien fondateur ${ }^{44}$, à tel point que le biologiste et anthropologue Fritz Lenz n'hésite pas à revendiquer pour ses travaux le prestigieux patronage de l'Athénien, " qui était tout autant eugéniste que philosophe 45 ». Richard Darré, ingénieur agronome, chef de l'Office central ss de la race et de la colonisation (sS Rasseund Siedlungshauptamt), puis ministre de l'agriculture du Reich, théoricien de l'idéologie Blut und Boden, s'intéresse lui aussi à Platon. Dans le travail qu'il lui consacre, Darré en fait le père de l'eugénisme, en reliant subtilement idéalisme et sélectionnisme. Comment, en effet, se prétendre théoricien des Idées si l'on n'érige pas l'Idée, qui est à la fois Forme et Norme, très haut au-dessus d'une réalité dévaluée. Qui dit idée, dit donc hiérarchie, et sélection : "C'est Platon qui a donné au mot idea son sens philosophique, c'est lui qui est devenu par sa doctrine le premier fondateur de l'idéalisme [...] [et] qui a attribué à l'empire de l'Idée une valeur absolue, dominant tout - et ce même Platon, en sa qualité d'idéaliste, fut amené à concevoir l'idée de sélection ${ }^{46}$. "

Hans Günther, quant à lui, rappelle dans quelle situation d'urgence et de péril historique Platon apparait : "Il faut se souvenir que, au temps de Platon, la noblesse attique, les Eupatrides [...] était en train de mourir et que cette noblesse d'ascendance raciale nordique s'était croisée bien des fois avec des souches étrangères depuis les origines de l'Attique ${ }^{47}$. " Le temps de Platon " fut une époque de décadence 48 " marquée par la "dénordification et [...] la dégénérescence raciale " provoquées par une guerre du Péloponnèse absurde et fratricide, qui provoqué une hémorragie $49 \mathrm{du}$ meilleur sang nordique, la décimation des grands blonds dolichocéphales de la Grèce nordique originelle : "À l'époque de Platon, les blonds devaient être devenus une minorité bien faible [...] la race dominante à l'époque, la race nordique, [ayant] dû frôler la disparition totale lors de cette guerre ${ }^{50}$."

44 Hildebrandt, Platon. Der Kampf des Geistes um die Macht, p. 396.

45 F. LENZ, Menschliche Erblichkeitslehre und Rassenhygiene. Band II. Menschliche Auslese und Rassenhygiene (Eugenik), Munich, Lehmanns Verlag, 1932, 593 p., p. 413.

46 R. DARRE, Der Neuadel aus Blut und Boden, München, JF Lehmann Verlag, 1930, 231 p.

47 GünTHER, Platon als Hüter des Lebens, p. 24.

48 Ibid., p. 14.

49 La hantise de l'hémorragie est récurrente dans le discours raciste nazi, volontiers catastrophiste, voire apocalyptique, pour souligner l'ampleur et l'urgence du péril racial. Dans son fameux discours du 18 février 1943 consécutif à la reddition de la VIe Armée à Stalingrad, Goebbels revient obsessionnellement sur la perte de précieux sang nordique que la bataille a induite.

50 GÜNTHER, Platon als Hüter des Lebens, p. 14. 
Les chefs naturels de la communauté athénienne, les hommes nordiques, ayant été décimés par la guerre, la capitale de l'Attique s'abandonne à une démocratie populiste et basse, qui exalte l'individu et perd de vue la fin naturelle de l'État : la communauté du peuple ${ }^{51}$. Platon s'assigne donc pour mission de redonner une caste dirigeante à la cité, "d'éduquer des Führer 52 " pour la polis athénienne, ce qui présuppose de disposer du matériel humain adéquat. On ne fait pas des chefs à partir de n’importe quelle matière humaine, " il faut d'abord trouver la matière dont on fait des dirigeants ". Pour redonner à Athènes une caste supérieure d'hommes nordiques revenus à la pureté et à la beauté originelle des paysans indogermaniques de l'Attique 53 , il faut favoriser les mariages entre égaux raciaux, afin de permettre la procréation d'enfants racialement purs 54 et imposer « l'élimination de tous les enfants difformes ou malades, l'extermination de tout ce qui est incapable de vivre 55 ".

Bien loin d'être un idéaliste naïf et innocent, Platon est ainsi exalté comme un représentant du réalisme le moins complaisant, le plus viril. Dans un opuscule intitulé L'État et la race, publié dès 1928, le platonicien Kurt Hildebrandt renverse la perspective habituellement adoptée sur Platon : "Platon n'était pas un utopiste, mais développait un réel savoir de la réalité et de la nécessité 56 » naturelles. Les mesures ségrégatives, sélectionnistes et eugénistes prônées dans La République sont citées en exemple de politique raciste accomplie 57 , des idées qui ont "imposé de douloureux sacrifices à l'idéalisme 58 » irresponsable de l'humanisme moderne et contemporain. Platon n'est pas cruel quand il donne de telles lois à sa cité idéale, il est simplement un esprit conséquent qui épouse sans hésiter les lois et les desseins de la nature : "Ses lois ont quelque chose de la loi naturelle " qu'elles respectent donc en interdisant toute atteinte contresélective au bon déroulement de la vie naturelle que la vie sociale ne doit venir obérer par aucun artifice.

Platon a "parfaitement compris que tout obstacle à ce que l'on désigne aujourd'hui comme une sélection naturelle " peut bien profiter à l'individu ainsi soigné, épargné, mais « nuit au peuple envisagé dans son ensemble ». Le bien-être, le bonheur ou

51 Günther n'a pas de mots assez durs pour fustiger la domination des démagogues et des sophistes, qui promeuvent un individualisme délétère et destructeur de la communauté traditionnelle : "Autant les premiers Grecs avaient considéré et évalué l'homme à partir de la communauté, autant les sophistes considéraient l'État du point de vue de l'individu et de ses désirs ", ibid., p. 15.

52 Ibid., p. 20.

53 Ibid., p. 30.

54 Ibid., p. 34.

55 Ibid., p. 33.

56 Hildebrandt, Staat und Rasse. Drei Vorträge, Veröffentlichungen der Schleswig-holsteinischen Universitätsgesellschaft, 19, Breslau, Ferdinand Hirt, 1928, 53 p., p. 37.

57 Ibid., p. 35.

58 Ibid., p. 36. 
la survie de l'individu importent peu au créateur d'État, au législateur et au Führer nordique : Platon « envisage toujours la totalité [du peuple], se détournant donc du cas individuel ", tant "la clémence [contre un individu dégénéré] serait apparue à Platon comme une cruauté contre le peuple envisagé comme tout 59 ", dure loi des nombres qui, pour le platonicien, comme pour les eugénistes, oppose le zéro individuel à l'infini de la masse. L'élimination sans complaisance (rücksichtslose Ausmerzung) du raté et du taré satisfait donc au principe politique holistique fondamental énoncé par le Parti nazi dès la rédaction du programme de 1920, et inlassablement ressassé depuis : Gemeinnutz geht vor Eigennutz, "l'utilité commune passe avant l'intérêt individuel ».

Une telle politique apparaît difficilement concevable à l'humaniste confit en miséricorde qui, myope, arrête son regard à l'individu et oublie de considérer ce qui donne sens, existence et vie à l'individu, son groupe, qu'un sujet difforme ou raté peut contaminer s'il est autorisé à vivre et à procréer : l'auteur réitère ici tous les arguments des partisans de l'eugénisme et de l'éradication du risque biologique par une politique de neutralisation (stérilisation) ou de destruction (élimination physique). Pour apaiser les peurs ou prévenir les objections des frileux humanistes de son temps, Günther, bon prince, précise cependant qu'il ne requiert pas l'impitoyable dureté des prescriptions de Platon. Ses solutions sont celles, civilisées, de l'eugénisme contemporain : Günther se contente donc de réclamer la "stérilisation 60 " des individus ratés en lieu et place de la peine de mort dont il prête l'exigence à Platon.

En irréductible ennemi de l'idéologie humaniste, compatissante et faible, des Lumières, Günther rappelle que les ennemis de Platon n'étaient autres que les sophistes, qui étaient "des hommes de race asiatique, comme nous l'enseigne la science raciale 61 ". Un manuel d'histoire de l'enseignement secondaire développe ces propos de Günther en affirmant que si les Grecs ont été réceptifs au discours individualiste des sophistes, c'est que leur sang était déjà gâté, en vertu de ce strict déterminisme qui conclut de la race à l'esprit :

La sophistique est donc complètement étrangère à la pensée nordique, et s'avère un rejeton de la race d'Asie mineure, qui a coutume d'opposer des concepts et de mettre en question des idées héritées, pour les détruire. L'influence de cette philosophie ne pouvait grandir que parce que les Grecs étaient devenus infidèles à leur origine. S’ils étaient demeurés un peuple de race pure, cet accent porté sur l'exceptionnalité de l'individu et de ses droits leur aurait été incompréhensible ${ }^{62}$.

62 W. Hohmann, W. Schiefer, Lehrbuch der Geschichte - Oberstufe - 1. Band, Frankfurtam-Main, Verlag Moritz Diesterweg, ca. 1940, 203 p., p. 64. 
Une partie de ce paragraphe extrait d'un manuel est un décalque littéral d'un passage de Hans Günther, copié au mot près sans être cité. Le passage plagié est tiré de l'ouvrage d'anthropologie raciale que Günther a consacré, en 1929, à l'histoire raciale des Grecs et des Romains : "La race d'Asie mineure a coutume de faire jouer des concepts les uns contre les autres, de critiquer de vieilles affirmations, de mettre en question, puis de détruire des conceptions héritées ${ }^{63}$. " Fidèle à sa symptomatologie raciale de toute œuvre culturelle, Günther considère la sophistique « totalement imprégnée d'esprit non-hellénique 64 ».

Les temps n'ayant guère changé, car les principes raciaux à l'œuvre et le combat qui les oppose sont les mêmes que dans l'Antiquité, les nouveaux ennemis de la race nordique sont 1789 et, significativement, le "sophiste Rousseau 65 ", digne rejeton d'une France mélangée et tout entière livrée à une idéologie égalitaire dont le projet "d'école unique 66 " du radical Herriot, antithèse absolue de l'éducation sélectionniste et élitaire de Platon, est un déplorable, navrant et révélateur symptôme. Contre l'égalitarisme aveugle de la modernité révolutionnaire qui proclame un inepte et imprudent " la même chose pour tous ", il faudrait revenir à un viril, nordique et platonicien "à chacun son dû 67 ", ce fameux Jedem das Seine qui résonne comme une bien sinistre anticipation de la maxime forgée sur le portail d'entrée du camp de concentration de Buchenwald.

Platon est donc, comme le dit Hildebrandt, un " maitre pour notre époque 68 ", car même la " biologie moderne aurait du mal à proposer des lois plus adaptées à la sélection des meilleurs que les lois de Platon 69 ». Quiconque veut édifier un État organique, c'est-à-dire, au sens strict, un État-corps qui repose à la fois sur "l'unité par le sang 70 » et sur une stricte hiérarchisation raciale et fonctionnelle de la communauté du peuple, doit suivre les préceptes de Platon. La lecture du grand philosophe nordique, prévient Hans Bogner, ne doit pas procéder d'une simple et inutile " hellénomanie esthétique 71 ", mais exprimer la ferme volonté de mettre en œuvre " une renaissance de notre existence » nationale, renaissance qui repose sur « l'héritage

63 H. GüNTHER, Rassengeschichte des Hellenischen und des Römischen Volkes, München, Lehmanns Verlag, 1929, 152 p., p. 60.

64 Ibid., p. 60. Sur l'interprétation raciologique du phénomène sophistique, cf. également A. RosenberG, Der Mythus des zwanzigsten Jahrbunderts, Munich, Hoheneichen-Verlag, $1935,712 \mathrm{p}$.

65 GÜNTHER, Platon als Hüter des Lebens, p. 68.

66 Ibid., p. 70. En français dans le texte.

67 Ibid., p. 71.

68 HiLDEBRAndt, Platon. Der Kampf des Geistes um die Macht, p. 243.

69 Ibid., p. 246

70 Ibid., pp. 246-247.

71 BOGNER, Platon im Unterricht, p. 9. 
divers et riche d'un passé de mille ans 72 ». Bogner se félicite que la Grèce soit à l'ordre du jour de l'Allemagne contemporaine :

La présence et la prédominance de l'hellénique dans l'Allemagne nouvelle s'impose à tout observateur ; que l'on pense seulement à nos nouveaux édifices, aux Jeux et à l'idée olympiques, au sport, à l'unité redécouverte du corps et de l'âme, à l'État désormais conçu comme institution d'éducation et de sélection des hommes, à nos arts plastiques. À l'occasion de manifestations publiques, la foncière parenté et affinité élective qui existe entre germanité et hellénité est souvent soulignée 73 .

Le combat de Platon fut sublime en ce qu'il fut voué à l'échec. Malgré les fermes injonctions du maitre de l'Académie, penseur d'un racisme hiérarchiste, sélectionniste et eugéniste, la Grèce fut érodée par des flots de sang allogène, accueilli dans un pays que la guerre civile avait laissé dans un état d'oliganthropie et de faiblesse qui ne lui permettait plus de résister à l'assaut de l'Asie.

L'esprit grec, tant qu'il était demeuré purement nordique, avait trouvé pour expression la philosophie de Platon. L'infiltration du sang asiatique entraîne une dégénérescence des corps et, corrélativement, une décadence des esprits qui signent le passage malheureux de l'hellénique à l'hellénistique, de l'exclusivisme de la cité-État au cosmopolitisme de l'empire alexandrin. Cette grécité à ce point dégénérée qu'elle n’en mérite plus le nom a pour expression idéelle non plus Platon, mais les Stoïciens.

La doctrine des stoïciens, dont l'école se fédère au IVe siècle, marque une rupture par rapport à la conception traditionnelle du monde et de l'homme que défendaient les anciens. La cosmologie grecque était celle d'un monde clos, hiérarchisé et finalisé, où tout être ou objet tendait vers son lieu naturel. De cette cosmologie procédait aisément une conception hiérarchique et inégalitaire de la cité des hommes où, comme dans le cosmos, chacun occupait une place qui lui était assignée par l'ordre objectif et inégalitaire de la nature, les deux ordres, naturel (cosmos) et civique (polis) se répondant par homologie de structure ${ }^{74}$. Au rebours de cette vision inégalitaire et plurale, les stoïciens ont défendu une conception unitaire, moniste, de l'humanité : contre la conception clivée et ségrégée d'une humanité finalement polygénique, l'école de la Stoa propose la vision monogénique et une d'une humanité solidaire, définie par l'égale présence de la raison en chacun de ses représentants 75 .

Pour les nazis, la Stoa, la tard-venue des écoles philosophiques grecques, est l'expression philosophique du crépuscule de la race : elle marque le passage du nordiquehellénique flamboyant à un hellénisme vespéral, antithèse de cette hellénité pure et

72

73

74

75

Ibid., p. 10

Ibid., p. 9.

Cf. J.-P. Vernant, Les origines de la pensée grecque, Paris, PUF, Quadrige, 1962, 129 p. et M. VILLEY, La formation de la pensée juridique moderne, Paris, Montchrétien, 1975, 718 p.

Cf. Epictète, Entretiens, III, 13 et Marc-Aurèle, Pensées pour moi-même, IV, 45. 
héroïque qui avait été tant bien que mal maintenue jusqu’à Platon et incarnée une dernière fois par l'Académicien. Produit de l'immigration asiatico-sémitique qui submerge Athènes, exsangue, à la fin du Ve siècle, et du mélange racial qu'elle induit, l'école stoïcienne promeut une doctrine égalitaire qui détruit tout sens de la hiérarchie raciale, comme le remarque en 1930 le raciologue gobinien Ludwig Schemann : "On a senti et dit depuis longtemps combien le sang a joué un rôle essentiel dans la fondation, puis dans le développement de l'école stö̈cienne. Celle-ci a surgi de cercles presque exclusivement sémitiques ${ }^{76}$. "

L'historien de l'Antiquité viennois Fritz Schachermeyr, inépuisable herméneute raciste de l'histoire grecque et romaine, le confirme et le dénonce avec véhémence :

L'hellénisme nous montre le peuple grec en pleine dissolution dans le cosmopolitisme, donc en pleine dénordification. Le produit le plus remarquable de l'hellénisme, la Stoa, va bien dans la même direction. Elle a été élaborée par des Sémites et des bâtards, pour devenir un pseudo idéal tout juste bon à donner des arguments aux apatrides et aux ennemis raciaux des époques ultérieures 77 .

Schachermeyr pousse la complaisance et l'acribie raciologiques jusqu'à recenser avec précision les origines géographiques, et donc les identités raciales, des chefs de l'école stoïcienne :

Parmi les scholarques qui se sont succédé jusqu’à Panaitios, il n’y en eut qu'un qui venait d'une ville au sang majoritairement grec [...]. Les autres venaient de Cilicie, de Chypre et de Babylone. Zénon, le fondateur, était issu de la ville sémitique de Kition, sur l'île de Chypre $[\ldots]^{78}$.

Dans un autre de ses textes, Schachermeyr attribue génériquement aux philosophes hellénistiques une " ascendance levantine 79 ». Max Pohlenz, grand spécialiste du Portique, et auteur d'ouvrages de référence sur le stoïcisme jusque dans les années 1970, parle, à propos du même Zénon, d'un " phénicien de plein sang 80 ", d'un Vollblutphöniker: "Nous rencontrons dans la doctrine stoïcienne bien des traits qui

76 L. Schemann, Die Rasse in den Geisteswissenschaften. Band II. Hauptepochen und Hauptvölker der Geschichte in ihrer Stellung zur Rasse, Munich, Lehmanns Verlag, 1930, 419 p., p. 145-146.

77 F. SCHACHERMEYR, «Die Aufgaben der alten Geschichte im Rahmen der Nordischen Weltgeschichte», in Vergangenheit und Gegenwart 23 (1933), p. 589-600, p. 599.

78 Ibid., n. 15, p. 599.

79 F. SCHACHERMEYR, Indogermanen und Orient. Ihre kulturelle und machtpolitische Auseinandersetzung im Altertum, Stuttgart, W. Kohlhammer Verlag, 1944, 635 p., p. 305.

80 M. Pohlenz, "Die Stoa - Geschichte einer geistigen Bewegung”, in H. Berve (dir.), Das Neue Bild der Antike. I - Hellas, Leipzig, Koehler und Amelang, 1942, 394 p., p. 354-368, p. 356. 
nous rappellent que ses fondateurs n'étaient pas des Grecs. "Inhelléniques, unhellenisch ${ }^{81}$, donc non nordiques, sont les idées et ce sang qui les a engendrées.

Nul esprit nordique n'aurait pu imaginer que tous les hommes pussent être considérés comme égaux et fussent appelés à être membres de l'universi generis humani socie-

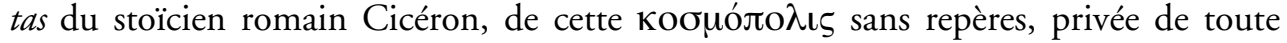
hiérarchie fondée sur l'axiologie du sang. Schachermeyr reprend ici un thème cher aux théoriciens de la race et du racisme qui, depuis Gobineau, attribuent toute idée égalitaire, individualiste et démocratique, à un sang gâté par le mélange, écrêté de toute qualité remarquable, un sang qui, donc, a perdu toute notion de son excellence et de la hiérarchie raciale qu'elle induit : un sang pur de tout mélange et conscient de sa valeur ne peut produire, par strict déterminisme biologique et simple consécution physiologique, que des idéaux élitaires, et non des idées égalitaires : ce réductionnisme ombrageux à la détermination biologique, cette réduction matérialiste de toute production culturelle, présente chez Gobineau, est reprise tout entière par les nationaux-socialistes. La Stoa nous est donc présentée et dénoncée par les raciologues et historiens nazis comme l'anti-Platon par excellence. Ludwig Schemann, disciple fribourgeois de Gobineau déjà cité, dénonce dans la Stoa, poison sémite, cette "idée cosmopolite et égalitaire 82 " qui constitue le cœur de sa doctrine.

Symptôme de la dégénérescence raciale grecque, la doctrine stoïcienne fut ensuite le puissant levier de la décadence romaine : après Cynocéphales (197 av. n.è.) et la conquête de la Grèce par les Romains, le stoïcisme fait en effet partie des dépouilles opimes remportées en butin par les vainqueurs. Livres, prisonniers de guerre et greaculi ramenés dans les fourgons des légions importent à Rome ce qui devient peu à peu la philosophie de référence des élites républicaines, puis impériales. Ces élites y trouvent un rigorisme compatible avec le mos majorum et une idéologie universaliste qui sied au mieux à l'impérialisme romain et à son projet d'hégémonie universelle. L'humanité unitaire des stoïciens a pour séjour un espace commun, appelé par la domination impériale romaine et l'unification de l'œkoumène qu'elle entraîne, à devenir une cosmopolis, cette civitas maxima unifiée qui réunisse la totalité de l'humanité sous un pouvoir et une juridiction identiques.

C'est sous l'inspiration de la doctrine stoïcienne que l'empereur Caracalla, guidé en outre par des intérêts fiscaux bien compris, accorde à tous les résidents de l'Empire la citoyenneté romaine en 212, une constitutio antoniana contre laquelle Alfred Rosenberg, dans son Mythe du XXe siècle, n'a pas de mots assez durs, l'érigeant en cause $\mathrm{du}$ " chaos racial romain ». Hans Günther, lui aussi, rappelle que "le stoïcisme a été considéré comme une des forces racialement destructrices de l'histoire romaine 83 » : la République nordique des origines, celle des Vieux Romains, strictement hiérarchique 
et inégalitaire, a été mise à mal par " l'individualisme " et " la citoyenneté cosmopolite 84 » que prône le stoïcisme. La faute dirimante de la Stoa est qu'elle «ne considérait que l'individu isolé d'un côté, et, de l'autre, la communauté des meilleurs issus de tous les peuples et de toutes les races, c'est-à-dire ni comme membre d'une lignée, d'un clan, d'un peuple ou d'une race ; la Stoa, par sa doctrine, levait toutes les barrières du sang 85 \%.

Comme la sophistique quelques siècles plus tôt, le stoïcisme est une déflagration intellectuelle qui annonce la Révolution de 1789. Schachermeyr affirme ainsi, en le déplorant, que " la conséquence de cette égalité fondamentale des hommes a été la conception d'une dignité de l'homme, de droits de l'homme, l'exigence de tolérance 86 ». Bref, par la faute du stoïcisme, " humanité rime désormais avec nivellement 87 ", et l'individualisme le plus délétère se trouve associé à l'universalisme le plus assassin.

Cette diabolique conjonction a entraîné la dissolution du grand corps de la race par l'infiltration et le mélange de principes allogènes qui ont fait apparaitre une nouvelle vision de l'homme et de son rapport au groupe. Jadis, quand le corps nordique des citoyens grecs était encore homogène et solidaire dans sa prime pureté, l'entr'appartenance mutuelle et spontanée de ses membres allait de soi : l'homogénéité du sang s'exprimait intellectuellement par une conception organiciste et holistique du corps politique, et l'individu ne prenait sens et ne revêtait dignité qu'en tant que membre du corps racial. Le mélange des sangs a brouillé cette conception unitaire du holos social conçu comme un corps racial. Déliée de toute appartenance à un sang et à un sol, la figure du cosmopolite advenue avec l'assomption de l'hellénisme ne peut plus se définir que comme individu atomique, comme monade isolée et dépourvue de tout lien à un groupe qui, de toute manière, n'existe tout simplement plus : la bâtardise hellénistique dissout la polis pour faire surgir l'individu ${ }^{88}$. Ainsi s'explique l'opposition entre la conception platonicienne de l'État, qui est sévèrement holistique, et la sensibilité individualiste des philosophies hellénistiques : «À la place de l'intérêt général, de l'inscription dans une communauté constituée par le sang, s'est imposée la domination effrénée de l'individu, du cosmopolite qui ne reconnaît d'autre lien que celui de sa raison. Le Grec n'a plus de patrie, son pays, c'est le monde ${ }^{89}$ [...]. "

Cet avènement de l'individu, "détaché de l'État et de son peuple, érigé en centre du monde 90 », le fractionnement du peuple grec « en une infinité d'atomes, dont

88 PohlenZ, "Die Stoa - Geschichte einer geistigen Bewegung”, p. 354-355.

89 F. Geyer, Rasse, Volk und Staat im Altertum, Leipzig und Berlin, B.G. Teubner, 1936, 172 p., p. 78.

Ibid., p. 106.

Ibid.

SCHACHERMEYR, Indogermanen und Orient, p. 307.

Ibid.

Ibid., p. 79. 
chacun n'existe que pour lui-même ", est l'œuvre de philosophes racialement dégénérés, comme "le fondateur de la Stoa, Zénon, un sémite hellénisé 91 ». Outre le principe racial, érigé en facteur explicatif, ce sont les arguments du débat contre les Lumières et l'individualisme jusnaturaliste moderne qui sont opposés à l'hellénisme, caricature, biologiquement dégénérée et intellectuellement décadente, de l'hellénité nordique des origines. Le XIXe siècle romantique allemand et ses épigones français, comme Maurice Barrès, opposent la cohérence naturelle, holistique d'une Gemeinschaft, celle de la naissance, de la terre, des morts et du sang, à la fragilité culturelle de la Gesellschaft, artéfact construit par la volonté contractante d'individus libres d'adhérer à un pacte social auquel ils sont supposés préexister : à la Gemeinschaft holistique du sang, de la détermination et de la nature s'oppose la Gesellschaft individualiste du choix, de la volonté et de la culture.

Un manuel de lycée nous explique d'ailleurs que toute l'ambition de Platon était la résurrection d'un principe holiste racial, d'une société conçue comme corps biologiquement solidaire : "Le véritable État n'est pas, aux yeux de Platon, composé d'individus [...]. Il est bien plutôt un organisme, une entité solidaire. Le citoyen n'est qu'un membre ou une part du tout et ne reçoit sa direction et sa définition que de la totalité ${ }^{92}$. "Un autre manuel lui consacre deux pages de même acabit et le présente en Don Quichotte de l'Attique, héros du sublime et " vain combat contre la décadence raciale 93 ".

Stigmatisée comme une philosophie de l'individualisme et de la rétraction, une doctrine du bonheur privé et de l'ataraxie dont jouit égoïstement le sage, loin de l'affairement du monde, le stoïcisme est une pensée de la dissolution de la cité, de la désagrégation du groupe politique et racial. Les stoïciens sont des destructeurs d'État parce que leur doctrine procède d'un sang destructeur de culture, destructeur d'État, barbare et anarchique : dans la typologie ternaire des races proposée par Hitler dans Mein Kampf, ces Orientaux et Sémites appartiennent à l'espèce des Kulturzerstörer, des destructeurs de culture, par opposition aux Kulturbegründer, aux créateurs de culture aryens ${ }^{94}$. Malgré cela, précisent tout de même Günther et Schemann, le stoïcisme a pu séduire de vieux Romains encore essentiellement nordiques, comme Sénèque et Marc-Aurèle, parce que ces hommes ne ressentaient plus de lien avec ce peuple romain racialement mélangé, et pouvaient donc souhaiter et concevoir se retirer dans le quantà-soi du bonheur privé, de la méditation du fatum et de l'ataraxie 95 : le déliement était ici un choix dicté par la médiocrité raciale du peuple romain dont il convenait de se séparer désormais.

91

92

93

94

95

\section{Ibid.}

HOHMANN, SCHIEFER, Lehrbuch der Geschichte, p. 67.

GeHL, Geschichte, p. 58 pour la citation, p. 58-60 sur Platon.

Cf. A. Hitler, Mein Kampf, p. 318.

GÜNTHER, Kleine Rassenkunde Europas, p. 146 sqq. et SCHEMAnn, Die Rasse in den Geisteswissenschaften, p. 146. 
Les stoïciens ont sapé et miné l'édifice inégalitaire, hiérarchique et holiste de la communauté aryenne traditionnelle par leur promotion coupable de l'individualisme. À partir du moment où les Stoïciens proclament que l'individu est source de toute valeur, les notions de peuple et de race perdent nécessairement la leur : la polis grecque et la civitas des vieux romains se trouvent dissoutes. La vigilance, selon Fritz Schachermeyr, s'impose ainsi au savant humaniste, au classiciste et à l'antiquisant, s'ils veulent, comme le souhaite tant l'auteur, conserver tout leur rôle et toute leur place dans le nouvel État national-socialiste. Ces spécialistes de l'Antiquité doivent être passeurs et médiateurs, mais seulement d'un matériau racialement valide et spirituellement pertinent : "On a jusqu'ici accepté tout ce qui venait de l'Antiquité comme une sorte de révélation sublime [...]. C'est ainsi que l'humaniste, qui était d'un côté le conservateur de l'esprit nordique le plus noble est devenu le médiateur de tout ce patrimoine spirituel anti-nordique ${ }^{96}$.

Seul un humanisme aveugle, une adoration béate de l'antique qui ne fait pas le départ du bon grain nordique et de l'ivraie asiatico-sémitique, a pu conserver à la culture ce "poison destructeur » qui a « dissout les peuples nordiques de l'Antiquité 97 " et qui demeure encore actif dans le monde contemporain. Toute Antiquité n'est pas bonne à prendre, et un humanisme qui se respecte présuppose donc un tri attentif dans les Humanités : la sélection (Auslese) aux fins d'épuration concerne donc autant les corps, dans les centres de l'opération T4 ou sur la rampe d'Auschwitz, que les âmes et lettres.

\begin{tabular}{l} 
Johann CHAPOUTOT \\
\hline Institut Historique Allemand de Paris \\
67 rue Pascal, F-75013 Paris. \\
johannchapoutot@hotmail.com
\end{tabular}

96 SCHACHERMEYR, "Die Aufgaben der alten Geschichte", p. 599.

97 Ibid. 